\title{
A Survey on Cloud Computing Service Models and Big Data Driven Networking
} Kiran Kumar S V N Madupu ${ }^{1}$

'Sr. PL SQL Dev / Data Base Specialist, System Soft Technologies, Herndon, VA

\section{ABSTRACT}

Cloud computing supplies the guarantee of big data implementation to little and also medium sized services. Big Data processing is executed with a programs paradigm called MapReduce. Usually, application of the MapReduce paradigm requires networked attached storage space and parallel handling. The computing demands of MapReduce programming are typically beyond what tiny and also average sized service have the ability to dedicate. In the paper, we briefly went over concerning cloud computing service versions as well as big data driven networking.

Keywords : Cloud Computing, Big Data, Data Mining

\section{INTRODUCTION}

BIG data is one of the "best" phrases being used today. Everyone is discussing big data, and it is believed that scientific research, service, sector, government, culture, etc. will undergo a detailed change with the impact of big data. Technically talking, the process of taking care of big data incorporates collection, storage, transport and also exploitation. It is no question that the collection, storage space and transportation stages are required forerunners for the utmost goal of exploitation through data analytics, which is the core of big data processing.

Counting on a data analytics viewpoint, we keep in mind that "big data" has happened defined by the four V's-- Volume, Velocity, Honesty, and Selection. It is thought that either all or any kind of one of them needs to be fulfilled for the classification of a trouble as a Big Data problem. Quantity indicates the size of the data, which could be too big to be handled by the present state of algorithms and/or systems. Velocity implies data are streaming at prices quicker than that can be handled by typical formulas and also systems. Sensors are quickly checking out and interacting streams of data. We are coming close to the world of evaluated self, which is presenting data that was not readily available hitherto. Veracity suggests that regardless of the data being offered, the high quality of data is still a major problem. That is, we can not think that with big data comes higher quality. As a matter of fact, with size comes top quality problems, which requires to be either tackled at the data pre-processing phase or by the learning formula. Variety is the most compelling of all V's as it exists data of various types and techniques for a given things in factor to consider.

Each of the V's is certainly not new. Artificial intelligence and data mining researchers have actually been taking on these concerns for years. How- ever, the emergence of Internet-based firms has tested most of the traditional process-oriented firms-- they now require to come to be knowledgebased business driven by data as opposed to by process. 
The objective of this short article is to share the authors' opinions about big data from their data analytics perspectives. The four writers bring quite various perspectives with various research experiences and also expertise, covering computational knowledge, machine learning, data mining as well as science, and also interdisciplinary re- search. Writers represent academia and also sector across four various continents. This variety combines a fascinating viewpoint as well as insurance coverage on checking out data analytics in the context of today's big data.

It is worth highlighting that this article does not mean to supply a comprehensive evaluation concerning the state-of-the-art of big data research, neither to supply a future big data research study program. The objective is to reveal the writers' individual viewpoints and also present their viewpoints of the future based on their sights. Because of this there will be always minimal indicatory argument or literary assistance, provided the swiftly changing landscape as well as substantial lag of academic study coverage. Indeed, lots of important issues as well as relevant techniques are not specifically covered in this article, and also are best entrusted to survey documents.

While all authors have contributed to the total paper, each author has actually concentrated on their particular specialities in the adhering to discus- sions. Zhou covers artificial intelligence, while Chawla brings a data mining and data scientific research viewpoint. Jin supplies a view from computational knowledge and meta-heuristic global optimization, and Williams draws upon a ma- chine understanding as well as data mining background used as an exercising data researcher and professional to sector worldwide.

Annually we have actually been observing a significant boost in our capability to collect knowledge from different sensing units, devices, in a number of formats, from freelance or attached applications. This large data has actually outmatched our capability to process, assess, keep and view these datasets. Take into consideration the data online. The net web pages indexed by Google were around 100 thousand in 1998, nevertheless quickly got to one billion in 2000 and also have actually already surpassed one trillion in 2008. In 2016 it is around 1.3 trillion.And will certainly be getting to roughly 2 trillion in year 2019. This rapid development of data is speed up by the remarkable rise in acceptance of social networking applications, like Facebook, Twitter, etc., that license users to form contents freely and amplify the already substantial internet volume. In addition, with smart phones are coming to be the sensory entranceway to get live understanding on individuals from completely various aspects, the enormous quantity of info that mobile provider will doubtless technique to boost our existence has actually significantly surpassed our past call data record based procedure for cost functions exclusively. It might be forewarned that Web of things (IoT) applications can raise the size of data to brand-new degree. Folks as well as gadgets from house occasional makers to cars and trucks, to buses, railway stations as well as airports, area system all loosely connected. Trillions of such linked elements can generate a huge knowledge cloud, as well as useful data must be uncovered from the details to assist improve quality of life as well as produce our globe a better place. As an example, when we often tend to increase each morning, so regarding optimize our commute time 
to figure as well as complete the optimization prior to we have a tendency to achieve office, the system needs to refine data from traffic, weather condition, construction, police activities to our schedule routines, as well as do deep optimization below the tight time constraints. used today by companies with a solid purchaser centre--retail, money relevant, document, and showcasing associations, to penetrate down right into their value-based information as well as choose evaluating, customer dispositions and product situating, affect on deals, consumer loyalty and also corporate benefits. With information mining, a retailer can make use of function of deal documents of customer gets to create items as well as developments to engage specific client segments. Some utilization of massive details remain in Study Evaluation, Corporate Monitoring, Fraud Detection, CRM.

\section{CLOUD COMPUTNG SERVICE MODELS}

Common deployment versions for cloud computing consist of platform as a solution ( $\mathrm{PaaS})$, software as a solution (SaaS), infrastructure as a solution (IaaS), as well as hardware as a solution (HaaS). Cloud release services can supply services that services would certainly or else not be able to manage. Companies can additionally utilize cloud deployment remedies as a test measure before taking on a brand-new application or modern technology company-wide.

There are a wide number of options for companies using the cloud for PaaS. System as a Solution is making use of cloud computing to supply platforms for the advancement as well as use of customized applications. The PaaS services include application design as well as development devices, application testing, versioning, combination, implementation, and hosting, state monitoring, and other relevant development tools. Organisations obtain cost financial savings utilizing Paas through standardization and also high usage of the cloudbased system across a variety of applications (Oracle, 2012). Various other advantages of using PaaS include decreasing risks by utilizing pretested modern technologies, promoting common services, enhancing software application safety, and decreasing ability needs required for brand-new systems development. As pertaining to big data, PaaS provides firms a platform for creating and using custom applications needed to analyze large quantities of unstructured data at a low cost and low risk in a secure environment.

Software as a service provides businesses with applications that are stored and run on virtual servers-- in the cloud. The business is not charged for hardware, only for the bandwidth for the time and number of users necessary. The main advantage of SaaS is that the solution allows businesses to shift the risks associated with software acquisition while moving IT from being reactive to proactive. Benefits of using SaaS are easier software administration, automatic updates and patch management, software compatibility across the business, easier collaboration, and global accessibility. Software as a Service provides companies analyzing big data proven software solutions for data analysis. The difference between SaaS and PaaS in this case is that SaaS is not going to provide a customized solution whereas $\mathrm{PaaS}$ will allow the company to develop a solution tailored to the company's needs.

In the IaaS model, a client business will pay on a peruse basis for use of equipment to support computing operations including storage, hardware, servers, and networking equipment. Infrastructure as a service is the cloud computing model receiving the most attention from the market, with an expectation of $25 \%$ of enterprises planning to adopt a service provider for IaaS. Services available to businesses through the IaaS model include disaster recovery, compute as a service, storage as a service, data center as a service, virtual desktop infrastructure, and cloud bursting, which is providing peak load capacity for variable processes (Cisco, 2009). Benefits of IaaS include increased financial flexibility, choice of services, business agility, cost-effective scalability, and increased security. 
While not as yet being used as extensively as PaaS, SaaS, or IaaS, HaaS is a cloud service based upon the model of time sharing on minicomputers and mainframes from the 1960s and 1970s. Time sharing developed into the practice of managed services. In a managed services situation, the managed service provider would remotely monitor and administer hardware located at a client's site as contracted. A problem with managed services was the necessity for some MSPs to provide hardware on-site for clients, the cost of which needed to be built into the MSP's cost. The HaaS model allows the customer to license the hardware directly from the service provider which alleviates the associated costs. Vendors in the HaaS arena include Google with its Chromebooks for Business, CharTec, and Equus.

\section{TYPES OF CLOUDS}

3 types of clouds exist-- the general public cloud, the personal cloud, and also the crossbreed cloud. A public cloud is the pay- as-you-go solutions formerly discussed available to the public. In a public cloud arrangement, a business does not have the core technology sources and solutions yet outsources these. A public cloud is taken into consideration to be an external cloud.

An exclusive cloud is interior data center of a business that is not available to the general public but uses cloud framework. In an exclusive cloud configuration, sources and also solutions are owned by the service, with the solutions accessible within business via the intranet. Considering that the technology is possessed and run by the company, this type of cloud is a lot more expensive than a public cloud, yet is additionally extra secure. An exclusive cloud is an interior cloud, residing inside the business's firewall software and also managed by the business.

When a company utilizes a crossbreed cloud, it utilizes a public cloud for some jobs as well as an exclusive cloud for various other jobs. When making use of a hybrid cloud design, a company will use the general public cloud to accelerate additional tasks that are unable to be easily run in the business's data center or on its exclusive cloud. A hybrid cloud permits a company to maintain vital, personal data as well as info within it firewall software while leveraging the public cloud for non-confidential data. The exclusive cloud section of the hybrid cloud is accessed by company staff members, both in the company and when traveling, and also is maintained by the inner innovation team. The personal cloud part of the crossbreed cloud is additionally accessed by the business workers however is kept by outside company. Each part of the hybrid cloud can connect to the various other part.

\section{WHICH CLOUD FOR YOUR DATA?}

The sort of cloud a company utilizes relies on the firm's requirements as well as sources. The general public cloud is thought about the least secure of the three kinds, with services and also resources able to be accessed online through methods taken on by the company. The communications protocols embraced by the supplier are not necessarily secure; the option of using secure or non-secure methods is up to the provides. The public cloud is additionally the least costly of the cloud types, with cost financial savings in the areas of information technology deployment, administration, as well as maintenance.

The personal cloud gives solutions to business staff members via an intranet. If mobile workers have the ability to access the exclusive cloud, the gain access to is typically through secure communication methods. All solutions and also sources provided are tailored to the requirements of the business, and also business has total control over the services as well as resources. As a result of the financial as well as personnels required to release, take care of, and also maintain the information technology resources as well as solutions supplied, the private cloud is one of the most pricey type of cloud. 
When a service utilizes a hybrid cloud, business has its core information technology sources and solutions as well as will certainly hold and offer the sources as well as solutions internal.

Non-critical services are outsourced and preserved on a public cloud. Normally, core infotech sources and also services are mission-critical and also are commonly confidential. Consequently, sources as well as solutions that require to be secure are hosted and preserved on the exclusive cloud, with the public cloud made use of for various other services as an expense conserving measure.

As gone over over, big data is commonly viewed as important for the success of the design optimization of facility systems. Much effort has been committed to the use of data to enhance the efficiency of metaheuristic optimization formulas for solving massive problems in the visibility of big amounts of uncertainties. It is thought that the boom in big data study can produce new opportunities along with impose brand-new difficulties to data driven optimization. Addressing the complying with inquiries can be main to converting the difficulties positioned by big data into possibilities.

First, just how can we effortlessly incorporate contemporary learning as well as optimization methods? Many advanced discovering strategies, such as semi-supervised learning [3], step-by-step understanding [5], active learning as well as deep understanding have been established over the past years. However, these techniques have rarely been made use of within optimization with few exceptions, as well as they are essential in obtaining domain name knowledge from a big quantity of heterogeneous as well as loud data. For optimization utilizing meta-heuristics, such knowledge is decisive in establishing a versatile and small issue depiction, developing effective search operators, creating top quality surrogates, and refining user choices in multiobjective optimization.

Second, exactly how can we formulate the optimization problem so that brand-new techniques devel- oped in big data research can be more efficiently leveraged? Conventional formulation of optimization issues consists of defining unbiased functions, choice variables as well as restraints. This works completely for little, well specified troubles. However, the formula- tion of complex issues is itself an iterative knowing procedure. The new approaches to data evaluation in big data can be of passion in simplifying the development of intricate optimization troubles. As an example, for surrogates to be utilized for rank forecast in population-based optimization, specific physical fitness forecast is less important than finding out the family member order of the candidate styles. Might it be additionally feasible to discover meta-decision variables that might be much more effective in assisting the search procedure than making use of the initial choice variables?

Third, exactly how do we visualize the highdimensional decision room as well as the highdimensional remedy room to understand the accomplished remedies and also decide? Exactly how can methods created in big data analytics be utilized in optimization?

Getting over these difficulties in a big data framework will certainly provide substantial breakthroughs to global optimization over the coming years.

\section{II.INDUSTRY, GOVERNMENT AND PEOPLE WITH BIG DATA}


We have actually offered above some of the technical obstacles for research study around the techniques affected and tested by big data. In the end, we likewise have to focus on the delivery of benefit and also end results within sector, company as well as government. Over the years, a lot of the modern technologies we have actually covered over, in artificial intelligence, data mining, and worldwide optimization, have located their means right into a selection of large applications. Yet what are the effects we see today in industry and government of big data and just how is this influencing as well as transforming society as well as just how might these adjustments affect our research study across all these self-controls? In this section we present a perspective on data analytics from the experiences in industry and federal government. The discussion is objectivefully offered as a point of view of the future in practice instead of presenting a scientifically extensive debate. We determine here areas where an emphasis from research might provide impact to market and also federal government.

It serves to show that over the past twenty years we have actually seen a period where culture has seen the mass collection of personal data by commercial interests and also government. As users, we have actually been enticed by substantial benefits to hand our data over to these organizations, and these organizations now house the big data that we have come to understand as the idea or the advertising and marketing regard to the moment.

Google, Apple as well as Facebook, together with many various other Internet business that exist today, give services ranging from the exploration of old close friends to the capacity to share our thoughts, personal details as well as day-to-day activities openly. With much of our e-mail, our journals and

also schedules, and our pictures and also ideas and personal tasks, currently hosted by Google, for example, there is remarkable chance to recognize and manage a whole-of-client sight on a massive range. Integrate that with our web logs, updates on our location as well as storage of our records on Google Drive, and also we start to comprehend the large range of the data gathered concerning each people, individually. These data can be utilized to much better target the services marketed to us, making use of an outstanding selection of algorithmic technologies to deliver new insights as well as understanding.

With each other, these group sourced data shops attract us to supply our personal data to the data collection agencies in return for the advanced services they use. The temptation is, certainly, remarkably appealing, shown by the sheer number of users in each of the expanding Net ecosystems.

The consumers that drive this data collection by these Net companies are not the users of the services however are, for example, business marketers and government services. The data is likewise provided to other organizations, purposefully and/or wrongly.

\section{BIG DATA DRIVEN NETWORKING}



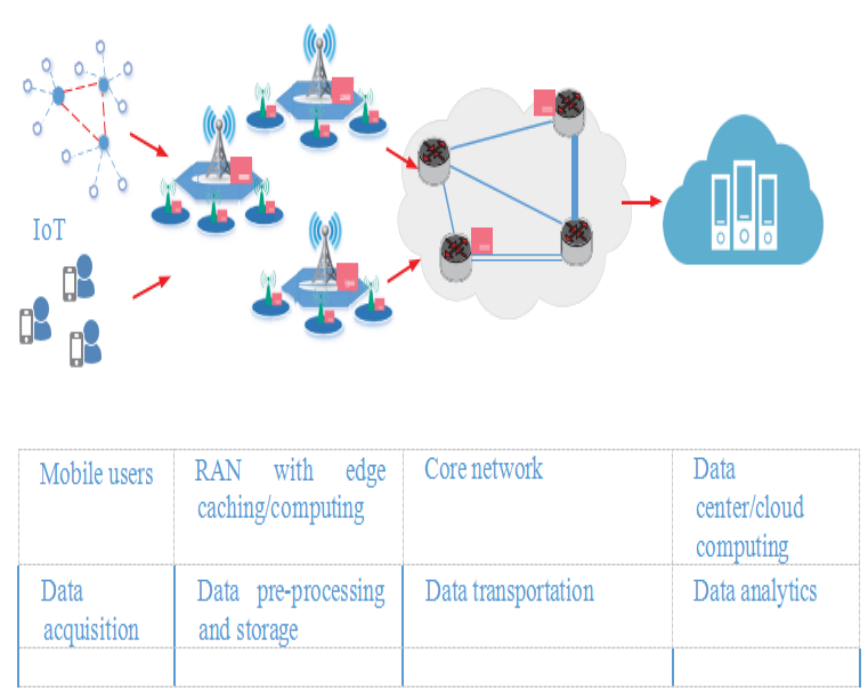

Figure 1 : Big data driven networking

B.Networking for Big Data

To better support big data and discover the big value, big data's attributes such as quantity, velocity, and also selection, must be efficiently fit by $5 G$ cordless networks. Firstly, big data quantity needs network capability to be considerably increased. The approaches to increasing cordless network capability primarily include spectrum development, spectrum effectiveness improvement, and network densification. The basic idea is to add more range resources, improve spectrum usage, and improve spatial spectrum reuse. Secondly, the velocity requires the data to be gathered, preprocessed, and delivered swiftly. Finally, the variety of data, which is likewise associated with different applications, need to be supported in data handling and transport by satisfying the matching demands. As a matter of fact, big data quantity is generally dealt with through network implementation, while rate and also selection are sustained via efficient network procedure, i.e., exactly how to utilize the infrastructure/resources to meet various needs. Fundamentally, it stipulations suitable networkbroad sources overall along the path from data source to data centers, to promote the big data processing chain. Because several big data applications (e.g., for power grid, economic evaluation, e-health) needs to be supported over the same framework simultaneously, the network-wide sources have to be shared efficiently.

An arising solution for service-oriented networking is network cutting, which creates various slices over the same physical framework, extending from gain access to domain to core network domain. Different pieces operate independently as well as share the same source pool. A network piece is a set of network sources for an offered application or make use of situation. It can be personalized to satisfy the matching end-to-end service requirement such as latency as well as integrity. The secret for network cutting is to dividers network-wide heterogenous resources for various pieces to sustain various use situations successfully. As a result, it is of significance yet testing to successfully use network resources while pleasing the solution needs of various applications or utilize instances.

With the development of network feature virtualization (N- FV), NFV can considerably promote network cutting. NFV enables network features to be produced in virtualized atmospheres, rather than on specialized equipment systems, therefore substantially improving network scalability and versatility. In core network, with NFV, a number of virtualized network features (e.g., web traffic splitting, data gathering, deep packet evaluation, and also firewall) that the data stream needs to undergo, can be made up, which is described as solution function chain (SFC). In other words, based upon the service need of application or utilize instance, an SFC can be created as needed, by composing various digital network features and identifying their implementation sequence. Different SFCs can be produced for unique applications/use cases and ingrained over the very same physical infrastructure. 
Furthermore, with virtualization, the capability of online features can be dynamically scaled out or in to adapt to the characteristics in networks and also solution needs. In radio access network (RAN), if NFV is supported, RAN cutting can be performed by developing digital RAN remarks, such as virtual BSs for different slices. Or else, network cutting is carried out based upon resource organizing. For example, at a specific RAN part such as a BS, with efficient scheduling, its sources can be alloted to and also shared by numerous slices. Furthermore, by meticulously alloting resources, the solution needs of various pieces can be pleased.

\section{CONCLUSION}

In big data handling chain, interaction, computing, and storage resources are needed at ideal areas along the path from data resource to data centers, to assist in data purchase, preprocessing, as well as storage. To provide for the rate and also selection, it signifies to provision tailored service-oriented end-to-end networking, which incorporates proper heterogenous resources and also functions, according to distinct demands from big data applications and utilize instances. In the paper, we quickly explained regarding cloud computing service designs as well as big data driven networking.

\section{REFERENCES}

[1]. A. K. Choudhary, J. A. Harding and also M. K. Tiwari, "Data Mining in Production: A Testimonial Based Upon the Sort Of Expertise", Journal of Intelligent Production, Quantity 20, Number 5, 501-521, 2008.

[2]. Arijay Chaudhry as well as Dr. P.S.Deshpande. Multidimensional Data Analysis and Data Mining, Black Book.
[3]. Smitha.T, Dr.V.Sundaram, "Understanding Discovery from Actual time data base making use of data mining strategy", International journal of Scientific Research and Magazine, (IJSRP) vol 2, concern 4, April 2012, ISSN 22503153, pp.74-76.

[4]. Smitha.T, Dr.V.Sundaram, "Comparative Study of Data Mining Algorithms for High Dimensional Data Evaluation"- published in International journal of Advancements in Engineering \& Modern Technology (IJAET) ISSN2231-1963 173 in vol 4, issue2, sept 2012 PP 15-20.

[5]. P. Zikopoulos, C. Eaton, D. deRoos, T. Deutsch, and also G. Lapis. IBM Understanding Big Data: Analytics for Venture Course Hadoop as well as Streaming Data. McGraw-Hill Business, Integrated, 2011.

[6]. S. M. Weiss and N. Indurkhya. Anticipating data mining: a functional overview. Morgan Kaufmann Publishers Inc., San Francisco, CA, USA, 1998.

[7]. J. Gama. Understanding Discovery from Data Streams. Chapman \& Hall/Crc Data Mining and Knowledge Discovery. Taylor \& Francis Group, 2010.

\section{Cite this article as :}

Kiran Kumar S V N Madupu, "A Survey on Cloud Computing Service Models and Big Data Driven Networking", International Journal of Scientific Research in Science and Technology (IJSRST), Online ISSN : 2395-602X, Print ISSN : 2395-6011, Volume 4 Issue 10, pp. 451-458, September-October 2018. Available at doi https://doi.org/10.32628/IJSRST207257 Journal URL : http://ijsrst.com/IJSRST207257 